\title{
Non-traditional drug targets in behavioural pharmacology
}

Behavioural Pharmacology 2010, 21:375-377

For most of its history, behavioural pharmacology has been concerned with a well-circumscribed set of drug targets, which consist, essentially, of processes involved in the life cycle of a relatively small set of neurotransmitters. The processes of interest include the metabolism, release and reuptake of neurotransmitters and, predominantly, their interactions with pre- and post-synaptic receptors; and the major systems of interest have been (in roughly the order in which they were established), acetylcholine, the monoamines (noradenaline, dopamine and serotonin), the inhibitory amino acid GABA, endogenous opioids, the excitatory amino acid glutamate, and - a more recent addition to the canon - endogenous cannabinoids. The vast majority of studies within behavioural pharmacology fall within this rubric.

Recently, however, the scope of behavioural pharmacology has broadened beyond this traditional focus to encompass a range of novel targets, many of them made possible by advances in molecular biology. This Special Issue of Behavioural Pharmacology was planned as a showcase for these alternative approaches, and the Editors deliberately did not specify what would and would not qualify for inclusion. We expected to see a rich variety of submissions, including, but by no means confined to, three of the major new areas to which behavioural pharmacologists are directing their research efforts: neuroprotection, drug actions on intracellular signalling systems, and interactions with the genome that influence structural processes in the central nervous system, including neurogenesis. We were not disappointed. This Special Issue contains important papers in all of these areas, and more. We were able to accept twenty submissions, including twelve review articles, most of which deal, in various ways, with aspects of neuroplasticity. We are confident that many of these reviews will come to be seen as essential reference points in the coming years, in their respective areas, and some of them will be highly influential. A common thread running through the reviews, and also apparent in the empirical studies, is the involvement of multiple mechanisms underlying the effects of the drugs discussed, and/or a multiplicity of behavioural and physiological outcomes, in contrast to the conventional impulse within traditional behavioural pharmacology towards ever more specific and discrete effects and mechanisms of action.

The Special Issue begins with two review papers that focus on the current hot topic of adult neurogenesis. Canales provides a detailed review of preclinical and 0955-8810 @ 2010 Wolters Kluwer Health | Lippincott Williams \& Wilkins clinical evidence that psychostimulants, as well as other drugs of abuse, disrupt memory and other cognitive processes that are thought to be dependent on the integrity of the hippocampus. This is linked to evidence that chronic drug administration also interferes with hippocampal neurogenesis and that these effects may be responsible for at least some of the deleterious effects of drug abuse on cognition. Figueiredo et al. present a focussed review of the behavioural pharmacology of the glucose-dependent insulinotropic polypeptide (GIP), which, like many compounds originally identified in gut tissues, is also expressed in many areas of the CNS. Evidence is reviewed for an involvement of GIP, and its receptor, in neuroplasticity and neurogenesis, with implications for epilepsy, Parkinson's disease and Alzheimer's disease.

Two further reviews focus on processes regulating gene expression. Morris et al. review the pharmacology of histone deacetylases (HDACs), as elucidated by the use of HDAC knockout mice and specific HDAC inhibitors. HDACs, through their location within the cell nucleus, modify chromatin architecture and directly mediate the changes in gene expression that occur during the course of development and in adult life. The review presents a state-of-the-art account of the neurobiology of HDACs, and their potential role in neuroplastic processes underlying learning and memory, affective disorders, and addiction. Stewart et al. review the current state of knowledge regarding another compound with a broad spectrum of action within the CNS, the neurosteroid vitamin D. The vitamin $\mathrm{D}$ receptor acts as a transcription factor to regulate the expression of a large number of genes, with consequences far broader than the traditional role of vitamin $\mathrm{D}$ in the regulation of calcium balance, and with implications for brain aging and cognitive decline, epilepsy, affective disorders and schizophrenia.

The next two reviews present very different approaches to the study of post-traumatic stress disorder (PTSD). Kaplan et al. review the similarities and co-morbidity of PTSD with traumatic brain injury (TBI), stimulated by the high incidence and coincidence of these disorders in returning US military personnel. Both conditions are characterized by structural abnormalities in hippocampus, amygdala and prefrontal cortex, associated with a dysregulation of brain-derived neurotrophic factor (BDNF). Since BDNF promotes neural regeneration and restores cellular connectivity, it is proposed that DOI: $10.1097 / F B P .0 b 013 e 32833 e 7923$ 
treatments that enhance BDNF-related signalling could have potential to restore cognitive and emotional function in TBI and PTSD. A second review by Pinna considers the mechanism by which specific serotonin uptake inhibiting antidepressant drugs (SSRIs) are effective in the treatment of PTSD. Based primarily on a model of prolonged social isolation in mice, convincing evidence is presented that, rather than the conventionally-assumed mechanism, serotonin uptake inhibition, these drugs act more potently to promote the biosynthesis of the neurosteroid allopregnanolone, which is coreleased by GABAergic and glumatergic neurons to modulate the responsiveness of GABA receptors. One important piece of evidence in support of this account of antidepressant action is the antidepressant drug tianeptine, which was originally reported to enhance, rather than inhibit serotonin uptake, and was subsequently found to act primarily to enhance allopregnanolone synthesis, with neuroprotective effects. A related empirical study by Chu et al. reports that tianeptine prevented the development of dependence on, and antinociceptive tolerance to, morphine, suggesting that these effects might involve the dystrophic remodelling of hippocampus and other limbic areas by chronic administration of drugs of abuse, as described in the opening review paper. A further empirical paper, by Frye et al. examines the mechanisms underlying the variable anxiolytic effects of hormone replacement therapies. The results suggest that these effects depend on the extent to which the exogenous steroids promote the metabolism of progesterone to allopregnanolone in the hippocampus.

Continuing the steroid theme, two review papers, by Estrada-Camarena et al. and Lukuge et al. discuss the antidepressant effects of estrogens. The first of these papers reviews the evidence that estrogens act as antidepressants and potentiate the effects of conventional antidepressants in various animal models, with effects dependent on a variety of factors including, inter alia, age, sex, treatment duration, and the specific estrogen used. These effects are discussed in relation to actions on monoamine systems, but also to the stimulation by estrogens of BDNF synthesis and neurogenesis. The second paper covers similar ground but focuses on recently-discovered membrane bound estrogen receptors (which differ in their cellular location from the classical nuclear estrogen receptors). The hypothesis is advanced that rapid antidepressant-like effects of estrogens may be mediated by membrane-bound estrogen receptors influencing intracellular signalling systems, and that this could represent a target for drug development.

Three more papers continue the discussion of the mechanism by which drugs influence affective processes. In a definitive review paper, Fornai elucidates the mechanisms underlying the complex pharmacology of lithium. It is proposed that, through a primary action of competition with the magnesium ion, lithium influences a host of enzymatic processes, but most significantly inhibits two enzymes, glycogen synthase kinase 3 beta and phosphatidylinositol phosphatase. This leads to downstream effects on a variety of intracellular signalling cascades, which also interconnect at several points with consequences for, on the one hand, mood and other behavioural effects, and on the other, neuroprotection and neurogenesis. Complex effects are also reported in an empirical study by Liebenberg $e t$ al. Stress stimulates the synthesis of nitrous oxide (NO), which enhances production of the intracellular messenger cyclic guanosine monophosphate (cGMP), and previous studies have reported antidepressant-like effects of NO synthesis and guanylyl cyclase inhibitors, and depressive effects of phosphodiesterase type 5 (PDE5) inhibitors, which block the degradation of cGMP. This study reports paradoxical antidepressant-like effects of PDE5 inhibitors, which are discussed in terms of dose-dependent interactions with monoamine and cholinergic systems. Finally, in a Short Report, Nishikawa $e t$ al. describe anxiolytic effects of a glycine transporter inhibitor. This study is of interest because, while glycine has been recognized as an inhibitory neurotransmitter since the early 1970s (almost as long as GABA), it stands outside the traditional canon because the lack of selective ligands has meant that glycine has received virtually no attention from behavioural pharmacologists.

The remaining review papers in this Special Issue describe novel targets in relation to addiction. Olive and Newton provide a comprehensive account, based mainly on studies in mice with targeted gene deletions, of the distinct roles of different isoenzymes of the protein kinase $\mathrm{C}$ (PKC) family of enzymes in regulating sensitivity to drugs of abuse. They identify several PKC isoenzymes that appear to be involved in processes underlying drug self-administration, and consider whether modulators of PKC isoenzymes might have a role in the treatment of addiction. The second paper in this series, by Bowers, takes as its target signal transduction at G-protein coupled receptors, which is modulated by a family of Activators of G-Protein Signaling (AGS). It has recently been established that one of these, AGS3, is upregulated by chronic treatment with commonly abused substances, but interestingly, the effects only become apparent after prolonged withdrawal, suggesting a potential role in relapse to drug use, and a potential strategy for the design of novel agents to combat relapse. The final review article, by Reissner and Kalivas addresses the same issue at a system level. They review evidence that addictive disorders are associated with dysregulation of glutamate homeostasis, based on functional changes in components of the homeostatic control mechanisms, including a cystine/glutamate exchanger and a glial glutamate transporter. They argue that these changes at the level of glutamate homeostatic mechanisms mediate long-term drug seeking, and that targeting them pharma- 
cologically restores the normal balance between synaptic and extra-synaptic glutamate levels, and also inhibits drugseeking behaviours.

We have already mentioned four of the eight empirical papers in this Special Issue. The remaining four papers are all concerned with compounds that have anti-oxidant properties, and consequently are neuroprotective by virtue of counteracting the neurodegenerative effects of oxidative stress. Zvejniece et al. demonstrate that the anti-ischaemic drug mildronate also has anticonvulsant activity and counteracts the hypnotic effect of ethanol, actions which are shown to be mediated by adrenergic and NO-dependent mechanisms. A potential role for mildronate in the treatment of seizures and alcohol intoxication is suggested. Organic preparations of the trace element selenium have been shown to have a variety of anti-oxidant, neuroprotective and beneficial behavioural effects. The second study, by Nogueira $e t a l$. reports that diphenyl diselenide protects against scopolamine-induced cognitive impairments, with implications for a potential therapeutic application in Alzheimer's disease. In the third paper, Khan et al. show that the destruction of dopamine neurons and the consequent impairment of locomotor behaviours in an MPTP model of Parkinson's disease are counteracted by chronic treatment with the anti-oxidant plant extract Pycnogenol. Finally, a Short Report by Park et al. presents evidence that the antioxidant EGCG, which is a major component of green tea, counteracts psychostimulant-induced increases in locomotor activity, potentially explaining why the caffeine in green tea has only minor stimulant effects.

The impetus for this Special Issue stemmed from the decision of the European Behavioural Pharmacology Society to dedicate its 2010 Workshop to the topic of 'Neurogenesis, drugs and psychiatric disorders'. The Special Issue ends with the Abstracts - over 40 in all of papers presented at that meeting, which will give those readers of Behavioural Pharmacology who are not themselves able to attend the meeting a foretaste of the future direction of this hugely important area of nontraditional research.

Paul Willner, Jack Bergman and Louk Vanderschuren July, 2010 